\title{
Performance Analysis of Different Combination of Mimo Antenna System using Digital Modulation
}

\author{
Anjali Juneja \\ M.Tech Scholar, Department of Electronics \& Communication Engineering, \\ Lakshmi Narain College of Technology, Indore, Madhya Pradesh, India
}

\begin{abstract}
How to cite this paper: Anjali Juneja "Performance Analysis of Different Combination of Mimo Antenna System using Digital Modulation" Published in International Journal of Trend in Scientific Research and Development (ijtsrd), ISSN: 24566470, Volume-3 | Issue-3 , April 2019, pp.532-534, URL: http://www.ijtsrd.co $\mathrm{m} /$ papers/ijtsrd 213 64.pdf

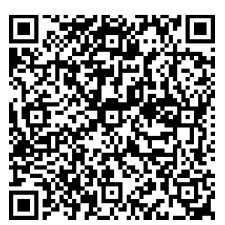

ABSTRACT

The wireless communication system like MIMO-OFDM (Multiple Input Multiple Output - Orthogonal Frequency Division Multiplexing), the transmitter and the receiver have to be synchronized to each other in order to make efficient transmission. In this paper, OFDM based on the orthogonality are proposed for timing synchronization for Rayleigh channel using different combination of antenna system. This work simulate by Matlab R2013a tool by using communication block, and result shown between BER verses SNR.

Copyright (C) 2019 by author(s) and International Journal of Trend in Scientific Research and Development Journal. This is an Open Access article distributed under the terms of the Creative Commons

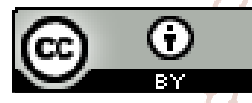
Attribution License (CC BY 4.0) (http://creativecommons.org/licenses/ by/4.0)

\section{INTRODUCTION}

The growing demand for mobile Internet and wireless multimedia applications has motivated the development of broadband wireless access technologies in recent years. Mobile WiMAX has enabled convergence of mobile and fixed broadband networks through a common wide-area radioaccess technology and flexible network architecture. Worldwide Interoperability for Microwave Access (WiMAX) has been considered as one of the key technologies that are capable of addressing the increasing demand for high-speed data communication.

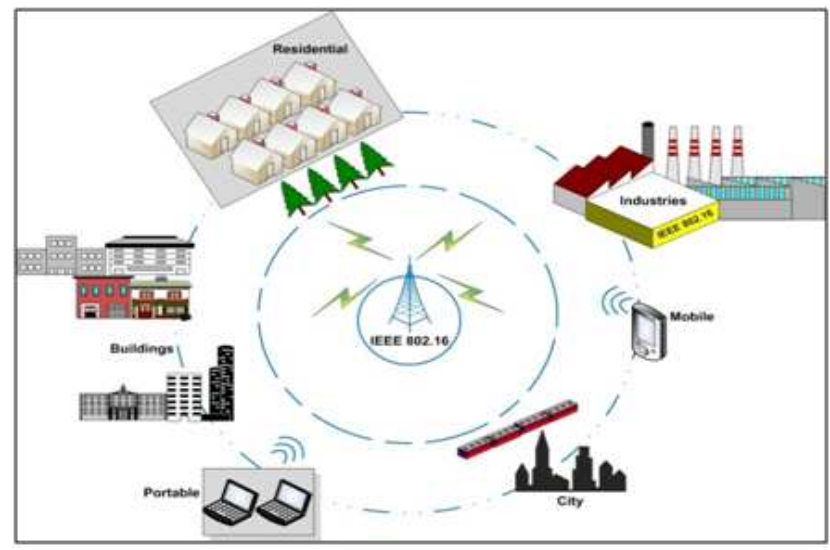

Fig.1: WiMAX Network System
WiMAX is an IEEE 802.16 standard based technology responsible for bringing the Broadband Wireless Access (BWA) to the world as an alternative to wired broadband. WiMAX is expected to have an explosive growth, as well as the Wi-Fi, but compared with the Wi-Fi WiMAX provides broadband connections in greater areas, measured in square kilometers, even with links not in line of sight.

The WiMAX Forum collaborated on the IEEE Std 802.16c2002 amendment to develop the system profiles for Wireless MAN-SC, it is forum helped developing IEEE Std 802.16Conformance Jan-2005, IEEE Std 802.16-Conformance Feb2005 and IEEE Std 802.16-Conformance Mar-2005 for a Protocol Implementation Conformance Statement (PICS) Performa, Test Suite Structure (TSS) and Test Purpose (TP) and Radio Conformance Test (RCT), respectively.

\section{Methodology}

Wireless MIMO channels have been recently attracting a great interest since they provide significant improvements in terms of spectral efficiency and reliability with respect to single input single-output (SISO) channels. The gains obtained by the deployment of multiple antennas at both sides of the link are the array gain, the diversity gain, and the multiplexing gain. The array gain is the improvement in signal-to-noise ratio (SNR) obtained by coherently 
International Journal of Trend in Scientific Research and Development (IJTSRD) @ www.ijtsrd.com eISSN: 2456-6470

combining the signals on multiple-transmit or multiplereceive dimensions while the diversity gain is the improvement in link reliability obtained by receiving replicas of the information signal through independently fading dimensions.

\section{Simulation Results}

Alamouti performs Monte-Carlo simulation and estimates Bit Error Rate (BER) of Alamouti Scheme over communication channel. The scheme presumes 2 transmit (Tx) and arbitrary number of receive $(\mathrm{Rx})$ elements. If $\mathrm{Rx}=1$ (one receive element) the alamouti is transformed to the order 2 transmit diversity scheme with Maximum Ratio Combining (MRC). The modulation format is MPSK with arbitrary order M which can be controlled by user.

In this work Mat lab R2013a is used to simulate and models the different problems for analysis and results. The simulations parameters are entered using the built graphic user interface (GUI) and contain:

1. Number of pair symbols from both Transmitter elements to be transmitted: $N$. This number should be at least 10 times more that the expected 1 /BER to provide low estimation error.

2. MPSK order $M$, must be a power of 2 . Since for $M=2$ (BPSK), $M=4$ (QPSK), $M=8$ (8-PSK), $M=16$ (16-PSK).

\section{Simulation Results for (2T1R/2T2R and 2T3R)} for BPSK Modulation

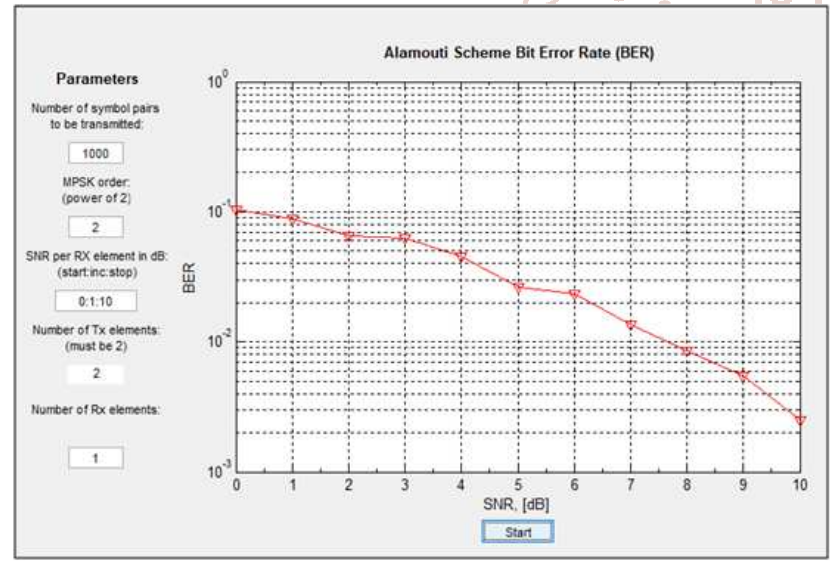

Fig. 2: Simulation Results for MISO (2T1R) for BPSK Modulation

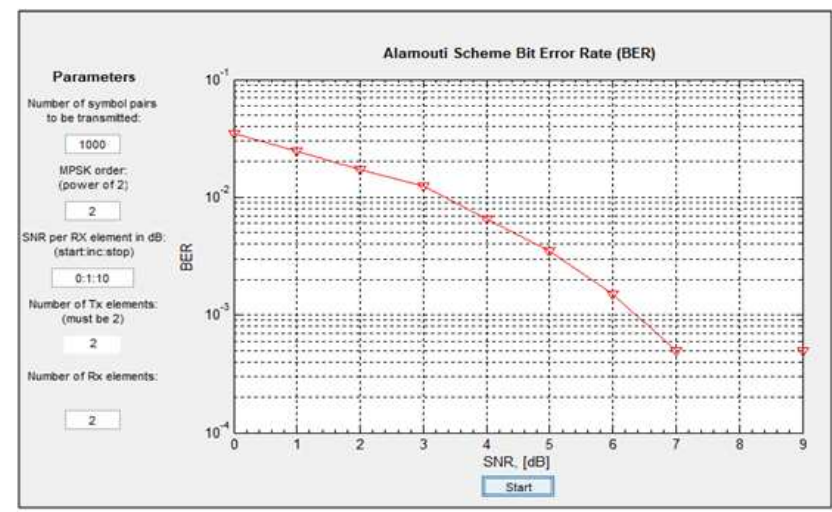

Fig. 3: Simulation Results for MIMO (2T2R) for BPSK Modulation

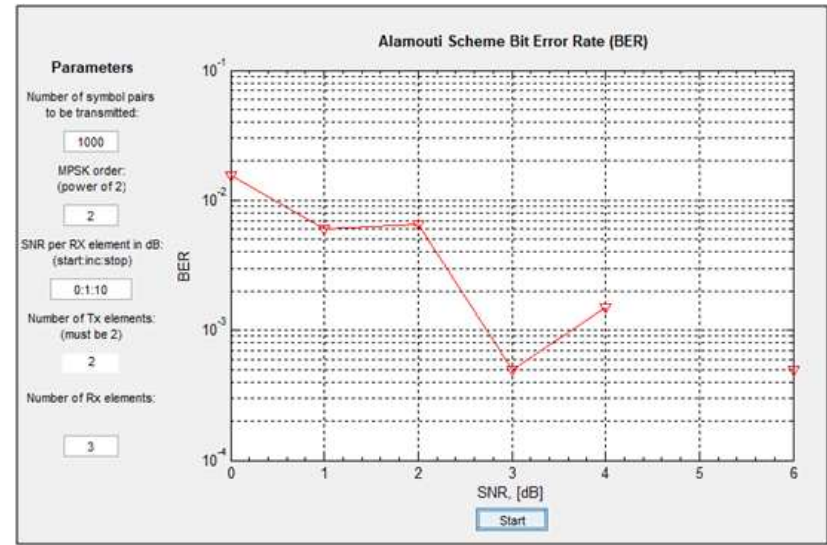

Fig. 4: Simulation Results for MIMO (2T3R) for BPSK Modulation

Table 1: Performance table for 2T1R/2T2R and 2T3R with BPSK

\begin{tabular}{|c|c|c|c|c|c|}
\hline S. No. & No. of Symbol & MPSK & TX-RX & BER & SNR \\
\hline 1 & 1000 & 2-PSK & 2T1R & $10^{-2}$ & 7.2 \\
\hline 2 & 1000 & 2-PSK & 2T2R & $10^{-2}$ & 3 \\
\hline 3 & 1000 & 2-PSK & 2T3R & $10^{-2}$ & 0.5 \\
\hline
\end{tabular}

\section{Conclusion}

In the above results based on linear modulation schemes (MPSK) - 2 PSK, with consider number of symbols is 1000 for $2 \mathrm{~T} 1 \mathrm{R}, 2 \mathrm{~T} 2 \mathrm{R}$ and $2 \mathrm{~T} 3 \mathrm{R}$ for the calculate SNR is $7.2 \mathrm{~dB}, 3 \mathrm{~dB}$ and $0.5 \mathrm{~dB}$ at $10^{-2} \mathrm{BER}$ respectively. The maximum SNR is $7.2 \mathrm{~dB}$ for $2 \mathrm{~T} 1 \mathrm{R}$ at $10^{-2}$, is better than $4.2 \mathrm{~dB}$ as compared to 2T2R.

\section{References}

[1] Ahmadi S., An overview of next-generation mobile WiMAX technology. IEEE Communications Magazine vol. 47, 2009, pp. 84-98.

[2] Andrews JG, Ghosh A, Muhamed R. Fundamentals of WiMAX: Understanding Broadband Wireless Networking (Prentice Hall Communications Engineering and Emerging Technologies Series). Prentice Hall PTR: Upper Saddle River, NJ, USA, 2007.

[3] IEEE. IEEE Standard for Local and Metropolitan Area Networks-Part 16: Air Interface for Fixed and Mobile Broadband Wireless Access Systems-Amendment for Physical and Medium Access Control Layers for Combined Fixed and Mobile Operation in Licensed Bands, IEEE, P802.16e/D7, 2005.

[4] Kamal Ali Alezabi, Abbas Jamalipour, Review Article, Authentication process enhancements in WiMAX networks, 2016, Security and Communication Networks, Volume 9, Issue 17, Pages 4703-4725.

[5] Ammar Ali Sahrab, MIMO-OFDM: Maximum Diversity Using Maximum Likelihood Detector, IEEE-2014.

[6] Mukesh Patidar, Rupesh dubey, Performance Analysis of WiMAX 802.16e Physical Layer Model, 2012 IEEE.

[7] S.M. Lalan Chowdhiury, P. Venkateswaran, Performance Analysis of WiMAX PHY, IEEE CASCOM Post Graduate Student Paper Conference 2010, Dept of electronics \& Tele-Communication Engg., Jadavpure University, Kollata, India. 
International Journal of Trend in Scientific Research and Development (IJTSRD) @ www.ijtsrd.com eISSN: 2456-6470

[8] G Kalyana Krishnan and V Umapathi Reddy, MIMO Communications - Motivation and a Practical Realization, IETE Technical Review. Vol. 25(6), 2014, pp. 203-213.

[9] Jian Li, Guoqing Liu, and Georgios B. Giannakis, Fellow, IEEE, Carrier Frequency Offset Estimation for OFDMBased WLANs, IEEE Signal Processing Letters, VOL. 8(3), 2001.
[10] IEEE 802.16-2006: IEEE Standard for Local and Metropolitan Area Networks, Air Interface for Fixed Broadband Wireless Access Systems.

[11] Biswajit Sahoo, Ravi Ranjan Prasad, BER Analysis of Mobile WiMAX System using LDPC Coding and MIMO System under Rayleigh Channel, International conference on Communication and Signal Processing, April 5-5, 2015.

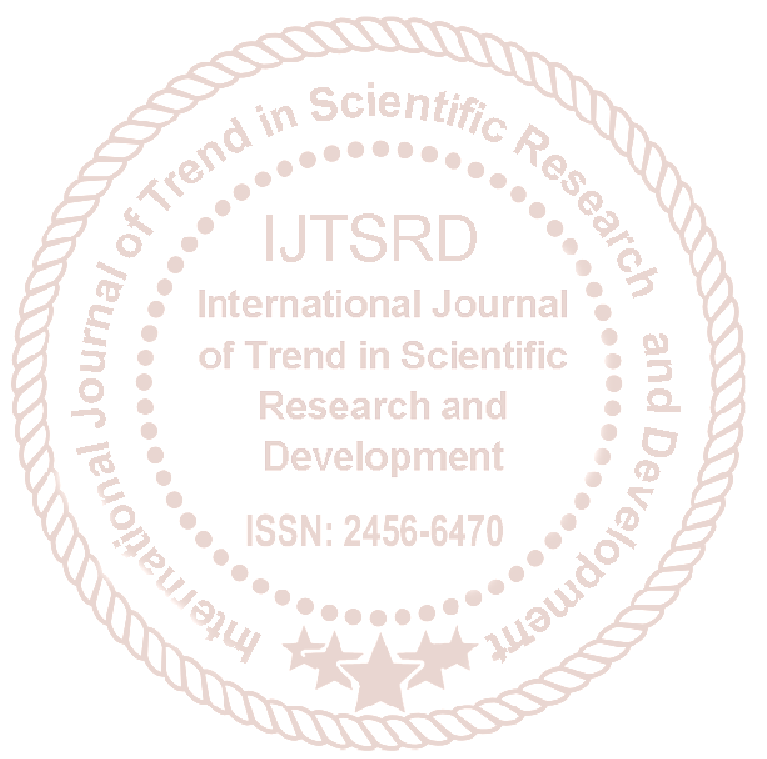

